\title{
The Excitatory Neuronal Network of Rat Layer 4 Barrel Cortex
}

\author{
Carl C. H. Petersen and Bert Sakmann \\ Department of Cell Physiology, Max-Planck-Institute for Medical Research, Heidelberg D-69120, Germany
}

\begin{abstract}
Sensory whiskers are mapped to rodent layer 4 somatosensory cortex as discrete units termed barrels, which can be visualized at high resolution in living brain slices. Both anatomical and physiological properties of the layer 4 neuronal network can thus be investigated in the context of the functional boundaries of this sensory map. Large-scale confinement of neuronal arbors to single barrels was suggested by restricted lateral diffusion of Dil across septa between barrels. Morphological analysis of dendritic and axonal arborizations of individual excitatory neurons showed that neuronal processes remain within the barrel of origin through polarization toward the center of the barrel. Functionally, the large-scale properties of the neuronal network were investigated through mapping the spatial extent of field EPSPs, which
\end{abstract}

were found to attenuate at barrel borders. This ensemble property of a layer 4 barrel was further investigated by analyzing the connectivity of pairs of excitatory neurons with respect to the locations of the somata. Approximately one-third of the excitatory neurons within the same barrel were synaptically coupled. At the septum between adjacent barrels the connectivity dropped rapidly, and very few connections were found between neurons located in adjacent barrels. Each layer 4 barrel is thus composed of an excitatory neuronal network, which to a first order approximation, acts independently of its neighbors.

Key words: neocortex; somatosensory cortex; barrel cortex; layer 4; synaptic transmission; EPSP; glutamate; neuronal network; dendritic morphology; axonal morphology
Sensory input is mapped to the neocortex such that closely related sensory stimuli are also close to each other spatially in the cortical representation. How individual neurons and neuronal circuits are functionally arranged within these maps is not well understood. This is in large part attributable to the difficulties of simultaneously studying both synaptic transmission between individual identified neurons and locating the neurons within the sensory map. The barrel field of the rodent somatosensory cortex provides a unique neocortical region for such investigations. Each sensory whisker is represented somatotopically in the large-scale anatomical structure of a barrel in layer 4 of the neocortex (Woolsey and Van der Loos, 1970). These anatomically defined structures have also been investigated electrophysiologically by extracellular unit recordings, and the location of the recording electrode within the barrel field is found to be homologous to the physiologically defined principal whisker (Welker, 1976; Simons, 1978; Armstrong-James and Fox, 1987). Thus, it is possible to relate the barrel pattern of the neocortex directly to the somatosensory map. Furthermore, barrellike structures are visible in the living brain slice preparation of somatosensory cortex (Agmon and Connors, 1991). The neuronal circuitry can thus be investigated at the level of individual neurons and their synaptic connections in the context of the functional boundaries of this sensory map.

The analysis of the excitatory neuronal network of layer 4 rat barrel cortex investigated in this study is thus a step to further our understanding of how the first layer of the neocortical circuitry may respond to input. In a recent study, Feldmeyer et al. (1999) described highly reliable local synaptic connections with large NMDA receptor components between excitatory neurons of layer 4 barrel cortex. In this study, we combine the investigation of the ensemble properties of barrels with the anatomy and physiology of individual neurons and pairs of neurons with the aim of understanding the collective properties of the underlying neuronal network. Whereas neurons lying inside the same barrel are often synaptically connected, neurons from neighboring barrels show low connectivity.

Received May 8, 2000; revised July 17, 2000; accepted July 25, 2000.

C.C.H.P. was supported by a Marie Curie fellowship from the European Commission. We thank Michael Brecht, Veronica Egger, and Dirk Feldmeyer for helpful discussions and comments on this manuscript.

Correspondence should be addressed to Carl C. H. Petersen, Department of Cell Physiology, Max-Planck-Institute for Medical Research, Jahnstrasse 29, Heidelberg D-69120, Germany. E-mail: petersen@mpimf-heidelberg.mpg.de.

Copyright (C) 2000 Society for Neuroscience $0270-6474 / 00 / 207579-08 \$ 15.00 / 0$
The functional data are reflected also in the underlying anatomy of the axonal and dendritic arbors, which are largely confined to individual barrels. Neighboring barrels thus appear to be only weakly connected to each other, and as a first order approximation we may consider each barrel as an isolated neuronal network. Such functionally independent neuronal networks of defined numbers of neurons and defined connectivity offer an opportunity to study the activity of physiologically relevant neuronal circuits in quantitative detail. As well as providing data on how sensory information is processed in the input layer of the neocortex, such a description of the functional architecture is also a prerequisite for understanding how reorganization of cortical representation occurs at a cellular level, for example after deprivation of sensory input (Diamond et al., 1994; Glazewski and Fox, 1996; Finnerty et al., 1999; Polley et al., 1999).

\section{MATERIALS AND METHODS}

Slice preparation. Thalamocortical slices of 250-300 $\mu \mathrm{m}$ thickness were prepared from anesthetized Wistar rats (13- to 15-d-old) following the description of Agmon and Connors (1991) with modifications by Feldmeyer et al. (1999). Slices were cut by a vibratome in ice-cold extracellular medium and were subsequently incubated at $35^{\circ} \mathrm{C}$ for $15-30$ min after slicing. The slices were then transferred to room temperature $\left(20-23^{\circ} \mathrm{C}\right)$ until required for analysis. Throughout the procedure slices were maintained in extracellular medium containing (in $\mathrm{mm}$ ): $125 \mathrm{NaCl}, 25$ $\mathrm{NaHCO}_{3}, 25$ glucose, $2.5 \mathrm{KCl}, 1.25 \mathrm{NaH}_{2} \mathrm{PO}_{4}, 2 \mathrm{CaCl}_{2}$, and $1 \mathrm{MgCl}_{2}$ bubbled with $95 \% \mathrm{O}_{2}$ and $5 \% \mathrm{CO}_{2}$.

Identification of barrels in living slices. The barrel subfield of somatosensory cortex is easily recognized in the thalamocortical slice preparation even by the naked eye. Under low-power light microscopy (Zeiss Axioskop fitted with a $2.5 \times$ lens) the dark band of laver 4 separates into barrels separated by lighter septal regions. To identify barrels at higher magnifications (Zeiss water-immersion lenses, $10 \times$ and $40 \times$ ), it is essential to increase contrast by closing the aperture. In this study only barrels that could be clearly distinguished at $40 \times$ with high-contrast apertures and further enhanced with a camera image processor (C2400; Hamamatsu, Tokyo, Japan) were used for further study. This selects for the most clearly delineated barrels and allows the detailed study of neurons in well defined regions of a single barrel. The barrels selected by these criteria did not necessarily lie in the posterior medial barrel subfield, and thus barrels with a wide range of diameters were investigated.

Identification of barrels by cytochrome C stain. Fixed slices were washed five times with PBS (100 mM sodium phosphate, $\mathrm{pH} 7.2$ ) over a period of $2 \mathrm{hr}$. Subsequently they were incubated at $35^{\circ} \mathrm{C}$ until clear staining of barrels were observed $(0.5-5 \mathrm{hr}$ ) in PBS containing (in $\mathrm{mg} / \mathrm{ml}$ ): 0.3 cytochrome $C, 0.3$ catalase, and 0.5 diaminobenzidine. Slices were subsequently washed five times with PBS over a period of $2 \mathrm{hr}$.

Quantification of barrel boundaries. Digital photographs of barrels in living or stained slices were taken using either a monochromatic camera 
(C2400; Hamamatsu) or a color CCD camera (Seescan LC100; INTAS, Göttingen, Germany). The photographs were converted into grayscale images and imported into IgorPro (Wavemetrics, Lake Oswego, OR) for quantitative analysis. Pixel intensities were measured as a function of position within layer 4. Maxima (bright) in this intensity function identify septal regions, which separate the low-intensity (dark) barrels. The intensity function was normalized such that the lowest brightness was represented by a value of zero and the maximal brightness by one. For quantification of the barrel boundary sigmoidal functions of the form $(a+b)$ $(1+\exp (c x+d)))$ were fitted, and the half-maximal brightness was chosen to indicate the edge of a barrel.

DiI injections. Barrels in layer 4 of somatosensory cortex of living slices were identified using high-contrast video microscopy. 1,1'-dioctadecyl3,3,3',3'-tetramethylindocarbocyanine perchlorate (DiI) (Molecular Probes, Eugene, OR) was dissolved in dimethyl formamide at $0.25 \%$. Small volumes of DiI were pressure-injected into the center of a barrel, and the amount injected was estimated by measuring the DiI signal within $1 \mathrm{~min}$ after injection. The mean diameter of the injections was found to be $43 \pm$ $6 \mu \mathrm{m}$. The slices were allowed to recover for several hours in extracellular medium before overnight fixation in $4 \%$ paraformaldehyde in PBS at $4^{\circ} \mathrm{C}$. Slices were subsequently washed five times in PBS and then incubated for 3 weeks at $35^{\circ} \mathrm{C}$. No obvious change in DiI fluorescence was observed between 2 and 3 weeks incubation, suggesting that the DiI diff usion had reached equilibrium within this period.

DiI labeling was subsequently analyzed both by standard epifluorescence and confocal fluorescence microscopy. Both methods gave equivalent results, however it was not possible to simultaneously observe barrel boundaries and DiI fluorescence under confocal microscopy, and thus only the epifluorescent measurements were used for further analysis. Epifluorescence was recorded by a CCD camera (Seescan LC100; INTAS) operating in a linear range allowing quantitation of the fluorescence signal. The fluorescence intensity distributions were analyzed by custom-written routines in IgorPro. Linear measurements of the spatial distribution of Dil fluorescence were made either parallel to layer 4 to quantify horizontal diffusion or vertically to quantify diffusion toward layer $2 / 3$ and the pia These fluorescence distributions were fitted by sigmoidal functions to quantify the half-maximal fluorescence values and also the $20-80 \%$ transition length as a measure of the steepness of the drop in fluorescence.

Biocytin staining and reconstruction. After staining of barrels by cytochrome $\mathrm{C}$ the slices were washed in PBS five times over a period of $2 \mathrm{hr}$ Endogenous peroxidases were then quenched by a 5 min incubation with $1 \% \mathrm{H}_{2} \mathrm{O}_{2}$. The slices were subsequently rinsed in PBS five times over a period of $2 \mathrm{hr}$. Slices were conjugated with avidin-biotinylated horseradish peroxidase following the manufacturer's instructions (ABC-Elite; Vector Laboratories). Slices were then washed five times over a period of $2 \mathrm{hr}$ with PBS, and subsequently biocytin-stained neurons were visualized under a reaction with $0.5 \mathrm{mg} / \mathrm{ml} \mathrm{DAB}$ and $0.01 \% \mathrm{H}_{2} \mathrm{O}_{2}$. When the neuronal processes were clearly visible, the reaction was stopped by washing with PBS. Finally the slices were mounted on slides using mowiol. Axonal and dendritic processes were subsequently reconstructed using Neurolucida software (Microbrightfield, Colchester, VT), and quantitative analysis of neuronal processes in relationship to barrel borders was performed by custom-written routines in IgorPro.

Field stimulation and recording. A glass microelectrode filled with extracellular solution with resistance of 3-4 M $\Omega$ was placed in the middle of a barrel identified under high-contrast video microscopy. Brief stimulating currents of $0.5 \mathrm{msec}$ duration were applied every $5 \mathrm{sec}$. The amplitude of the current injection (range, 15-50 $\mu \mathrm{A}$ ) was chosen to give a clearly identifiable field EPSP (fEPSP) (amplitude range, $0.3-0.7 \mathrm{mV}$ ) as measured at a recording site within the same barrel as the stimulating electrode. The recording electrode was also a glass microelectrode filled with extracellular solution with resistance of 5-6 $\mathrm{M} \Omega$, and voltage changes were monitored using an Axopatch 200B amplifier. The recording electrode was subsequently moved over a range of positions crossing the barrel boundary between the stimulated barrel and the nearest neighbor several times, and fEPSPs were compared at different locations evoked by identical stimuli.

Whole-cell recordings from identified layer 4 neurons. Layer 4 neurons were identified using infrared differential interference contrast microscopy. Excitatory neurons of layer 4 have round somata of $\sim 10 \mu \mathrm{m}$ diameter and often appear to be clustered. Slices were continuously perfused with extracellular medium bubbled with $95 \% \mathrm{O}_{2}$ and $5 \% \mathrm{CO}_{2}$. All experiments were performed at $35^{\circ} \mathrm{C}$. Whole-cell recordings were established using pipettes with resistances of $5 \mathrm{M} \Omega$ filled with a solution containing (mM) 105 potassium gluconate, $30 \mathrm{KCl}, 10 \mathrm{HEPES}, 10$ phosphocreatine, 4 MgATP, and $0.3 \mathrm{Na}_{3}$ GTP (adjusted to $\mathrm{pH} 7.3$ with KOH). Whole-cell electrophysiological measurements were made with Axoclamp 2A, Axopatch 200A, Axopatch 200B, or EPC-7 amplifiers. Biocytin $(2 \mathrm{mg} / \mathrm{ml})$ was routinely included in the intracellular solution to allow the morphology of the neurons to be more closely analyzed. Excitatory neurons were further identified by regular action potential firing pattern in response to continuous current injection; broad action potentials (half-widths of over $1 \mathrm{msec}$ ) and initial axonal segments directed toward deeper cortical layers.

Loose-patch recordings. Using the same pipettes as for whole-cell recordings except filled with extracellular solution and without the need to change pipette for each cell, it is possible by gentle suction $(10 \mathrm{mbar})$ to form electrically tight seals of $\sim 50 \mathrm{M} \Omega$ resistance between pipette and an identified neuron. In such a configuration it is possible to evoke action potentials by injection of $5 \mathrm{msec}$ duration current pulses with a threshold of $\sim 5 \mathrm{nA}$. The evoked action potentials are recorded as short duration depolarizations with amplitudes of $\sim 10 \mathrm{mV}$. Because of the requirements of strong current injections, only the Axoclamp $2 \mathrm{~A}$ amplifier was used for this recording configuration. The loose-patch configuration does not allow the analysis of the action potential firing pattern, giving rise to some uncertainty concerning the neurotransmitter identity of a presynaptic neuron, although this can often be judged from the appearance of the cell under IR-DIC. Thus, to isolate EPSPs, $10 \mu \mathrm{M}$ bicuculline was included in the extracellular medium. To test whether the loose-patch technique provides equivalent results to whole-cell recordings, we compared EPSPs evoked by loose-patch stimulation to EPSPs evoked by stimulation from the whole-cell recording configuration for the same cell pairs. In each case where loose-patch stimulation evoked responses in the postsynaptic neuron so did the whole-cell configuration. The amplitude of connections identified in loose-patch mode did not change significantly in the whole-cell configuration (ratio of EPSP evoked by loose-patch vs whole-cell action potentials was $1.1 \pm 0.2 ; n=9$ cell pairs). Thus, loose-patch stimulation correctly identifies connected neurons and quantitatively provides results comparable to whole-cell recordings for the EPSP amplitude.

\section{RESULTS}

\section{Neurons can be identified within a specific region of a barrel}

Each whisker is uniquely represented in the somatosensory cortex by a barrel-like structure as described by Woolsey and Van der Loos (1970). Although originally identified by histological methods, the barrel cortex of rats is equally obvious in living brain slices under bright-field illumination (Agmon and Connors, 1991). Regions of the neocortex that appear dark in bright-field microscopy are also heavily stained for cytochrome C oxidase (Fig. 1), which is one of the staining methods to identify barrels (Wong-Riley and Welt, 1980). To make a quantitative comparison of the structures visualized by the two different approaches, photographs of a slice under bright-field illumination were taken first, and then the slice was stained for cytochrome $\mathrm{C}$ oxidase. The color images (Fig. 1A2, $B 2$ ) were converted to gray scale, and the changes in the brightness of pixels parallel to layer 4 were plotted across the same region identified in both images. The maxima in the light intensity function represent the bright regions separating the barrels. These septa occur at identical positions in both techniques for identifying barrels. The structure visible by bright-field microscopy thus appears to be entirely equivalent to the one identified by cytochrome C staining.

Identification of barrels in bright-field microscopy raises the possibility that we can make measurements from a specific region of an individual barrel in layer 4 of living brain slices. Through use of video-enhanced high-contrast microscopy, barrel borders can be identified at high magnification using $10 \times$ (Fig. $2 A$ ) and $40 \times$ water-immersion lenses (Fig. $2 B$ ). This allows whole-cell recordings to be made from neurons very close to barrel borders (Fig. $2 A, B)$. Excitatory neurons were identified by small round somata often appearing in clusters and regular firing patterns with broad action potentials. Biocytin was included in the pipette solution, allowing the structure of the neuronal processes to be reconstructed later (Fig. 2C,D). Double staining for biocytin and cytochrome $\mathrm{C}$ reveals that the neuron, from which we recorded, indeed was located at the edge of the barrel (Fig. 2C,D). The data in Figures 1 and 2 indicate that the functional architecture of barrels can be studied at high resolution and correlated with the physiological and anatomical analysis of individual neurons.

\section{Axonal and dendritic arbors are confined to a single barrel}

To define the anatomy of layer 4 barrels we investigated the extent of the axonal and dendritic arborizations at a large-scale level and at the level of individual neurons. One method for tracing overall membrane structure is to measure the diffusion of fluorescent lipophilic molecules. We made microinjections of DiI into individual barrels of 10 slices and allowed the compound to diffuse in the cell membranes (Fig. $3 A$ ). Strong DiI labeling was observed to spread throughout the injected barrel and the associated vertical column of neocortex, but even after 3 weeks of diffusion, fluorescence signals were only weak in neighboring barrels. There was a 
A1

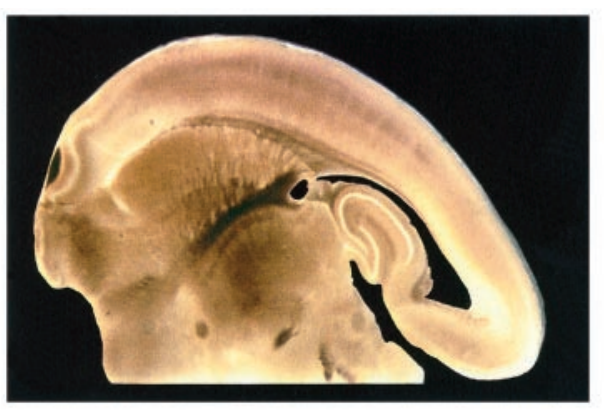

$\mathrm{A} 2$
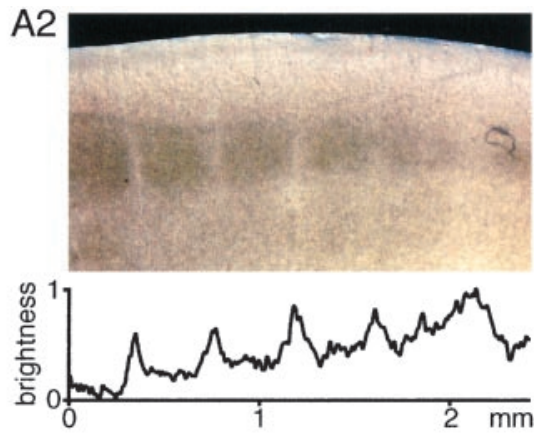

B1

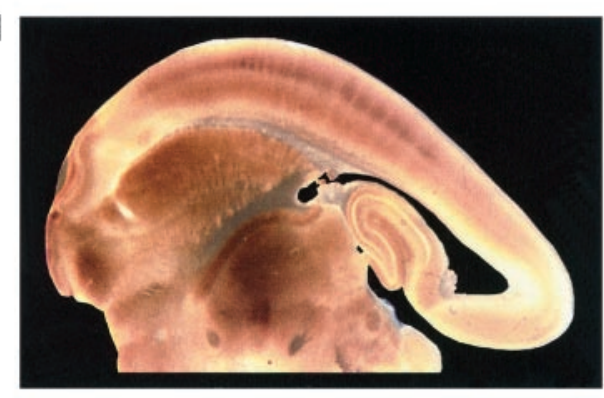

\section{B2}
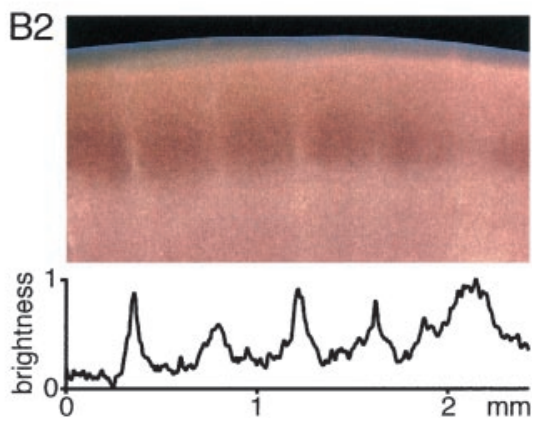

Figure 1. Barrels in layer 4 of somatosensory cortex identified by bright-field microscopy correspond to barrels defined by cytochrome $\mathrm{C}$ staining. $A$, Barrels can be visualized in brain slices of rat somatosensory neocortex using bright-field microscopy. A subset of the barrels are shown at higher magnification (A2), and the normalized brightness of pixels is quantified across the barrels in layer 4 . The dark barrels are observed to be clearly separated by light septa. $B$, The same slice as shown above was subsequently stained for cytochrome $\mathrm{C}$, which has been used to define barrels in previous work. A very similar pattern of dark regions separated by light septa within layer 4 of the neocortex is observed. The quantitative analysis of the normalized pixel brightness across the barrels $(B 2)$ indicates that there is an excellent correspondence between barrels defined by bright-field microscopy and barrels defined by cytochrome $\mathrm{C}$ staining.
A

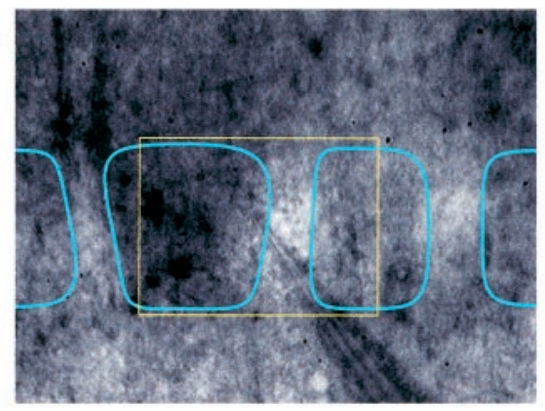

C

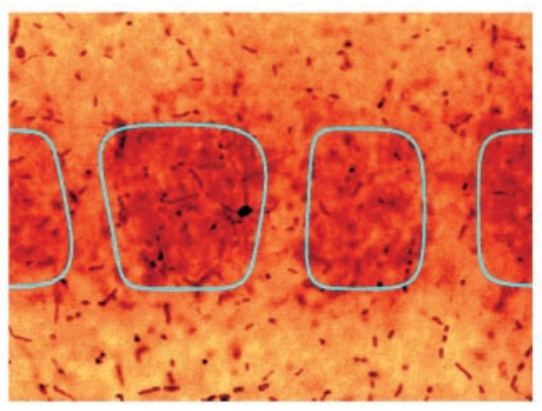

B

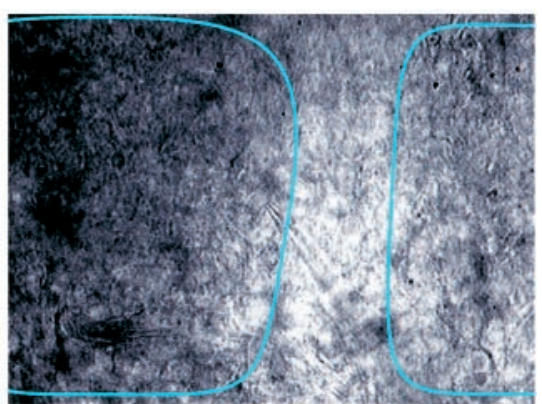

D

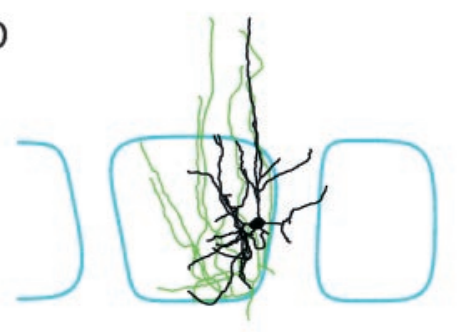

L2/3

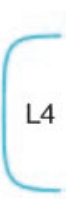

L5

Figure 2. Whole-cell recordings from identified neurons in a specific region of a barrel. Barrels within layer 4 can by visualized at high magnification by enhancing contrast through video microscopy, allowing whole-cell recordings to be made from a given region of a barrel. The neuron in this example is located on the right-hand of the barrel as seen with $10 \times(A)$ and $40 \times(B)$ water immersion lenses. During the electrophysiological recording the cell is filled with biocytin, allowing the neuron to be visualized later in conjunction with a cytochrome $\mathrm{C}$ stain $(C)$. These stains confirm the location of the neuron within the barrel and allow the axonal (green) and dendritic (black) arborizations to be reconstructed $(D)$ in relationship to the barrel boundaries (cyan). Scale bar: $A, C, D, 100 \mu \mathrm{m} ; B, 50 \mu \mathrm{m}$.

clear correlation between the width of the barrel and the spread of the DiI signal (Fig. 3B), suggesting that the DiI diff usion is laterally restricted to a single barrel. Equally the fluorescence intensity showed a sharp decrease at the barrel boundary compared to the longer vertical spread into layer $2 / 3$ (Fig. $3 C$ ). These results are in complete agreement with a recent study of biocytin uptake and diffusion after small extracellular injections into layer 4 barrel cortex in vivo, which also demonstrated labeling confined to the injected barrel (Kim and Ebner, 1999).

These DiI fluorescence results suggest that axonal and dendritic arbors in layer 4 should be confined to individual barrels. To investigate this at a cellular level, excitatory neurons located within a barrel were filled with biocytin. The axonal and dendritic structure of the labeled neurons was then reconstructed and correlated to the large-scale barrel morphology revealed by cytochrome $\mathrm{C}$ stains (Fig. 4A). The axons and dendrites of these neurons are indeed largely confined to the home barrel-the barrel where the soma is located. The analysis of 19 reconstructed excitatory neurons (some of which were in the middle of a barrel and some near the edge) show that $91 \%$ of axonal and $97 \%$ of dendritic length in layer 4 remains within the home barrel (Fig. 4B). To account for such a high degree of axonal and dendritic confinement to a single barrel, neurons located close to the border of a barrel have polarized axonal and dendritic arbors directed toward the center of the barrel, whereas neurons located in the middle of a barrel are less polarized (Fig. 4C). These results are in excellent agreement with previous descriptions of Golgi-impregnated layer 4 neurons, which showed that only $5 \%$ of neurons with somata located in a particular barrel have dendrites spanning additional barrels (Woolsey et al., 1975). Previous studies have also suggested that the axonal arborizations within layer 4 of barrel neurons are largely restricted to single barrels (Harris and Woolsey, 1983; Lübke et al., 2000). 
Figure 3. DiI diffusion is confined to the injected barrel. $A$, The layer 4 barrels are still visible in these bright-field micrographs ( $A 1$, $A 2$ ) of a slice that has been microinjected with DiI, fixed in paraformaldehyde, and incubated at $35^{\circ} \mathrm{C}$ for 3 weeks to allow DiI diff usion. The DiI injection was confined to a diameter of $50 \mu \mathrm{m}$ in the center of the middle barrel outlined in cyan (A2). DiI fluorescence images of the same slice $(A 3, A 4)$ indicate that although the DiI has spread vertically toward the pia, the diffusion of DiI in the horizontal direction is confined to remain within the barrel boundary. Scale bar, 100 $\mu \mathrm{m}$. $B$, The width at half-maximal intensity of the DiI fluorescence is well correlated to the width of the injected barrel (black line shows best linear fit by least squares method). $C$, The fluorescence intensity versus distance in the horizontal (within layer 4) or vertical (up toward the pia) was fitted by a sigmoidal function and the transition distance from $80 \%$ maximal to $20 \%$ maximal intensity is shown. At the barrel/septal boundary there is an abrupt decrease in DiI fluorescence, whereas the vertical spread decays slowly.
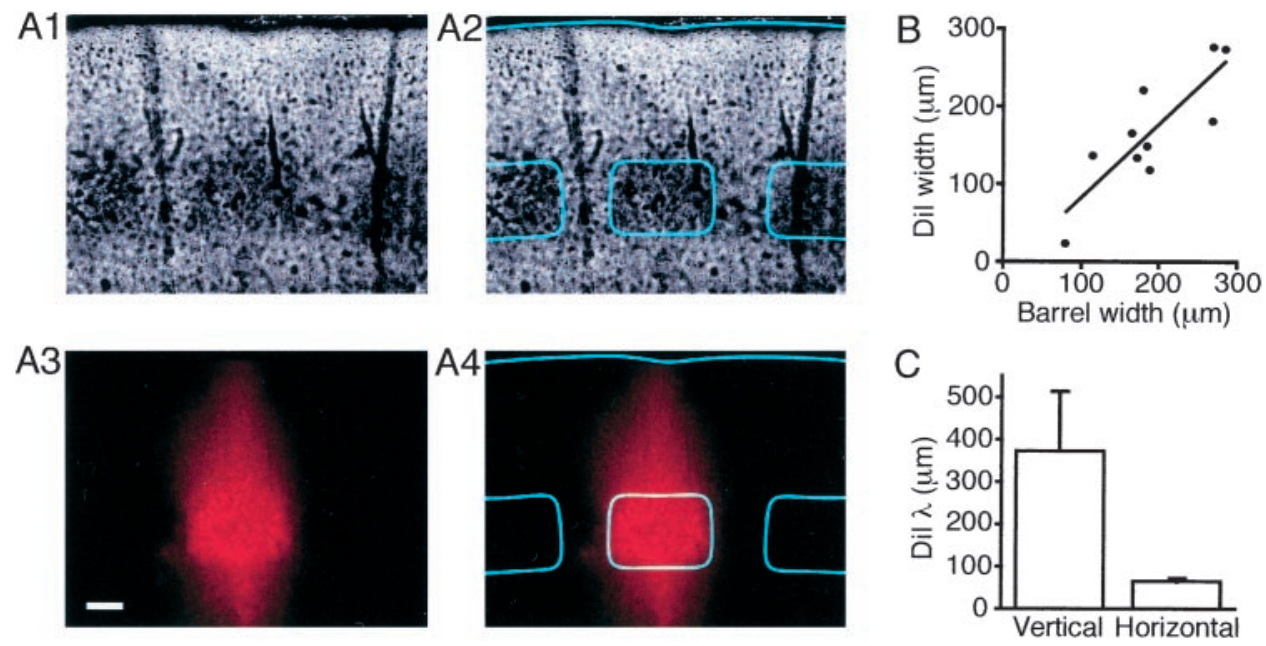

Figure 4. Axonal and dendritic processes are confined to the home barrel. $A$, An example of a reconstructed axon (green) and dendrite (black) of an excitatory spiny stellate neuron found at the right edge of a layer 4 barrel (outlined in cyan). Neuronal process are largely confined to the home barrel. Neurons with somata close to the edge of a barrel have axonal and dendritic trees oriented toward the center of the barrel. Scale bar, $100 \mu \mathrm{m}$. B, Quantification of the fraction of axonal (hatched bars) and dendritic (open bars) length within layer 4 found in the barrel in which the soma is also located (the home barrel), in the septa between barrels and in the adjacent barrels. Within layer 4 very little axonal and dendritic length is found outside of the home barrel. $C$, The polarization of axonal (open circles) and dendritic (filled squares) arbors within layer 4 is related to its location in the barrel. The $y$-axis is normalized so that a neuron that had its entire arbor to the left of the soma would be plotted at zero, whereas a neuron possessing only arborizations to its right would be plotted at unity. The $x$-axis is normalized for each barrel such that the left border of the barrel is represented as the left extreme, and the right border of the barrel is also the right extreme of the graph. The graph shows quantitatively that neurons are polarized with respect to their position in the barrel such that their processes avoid entering neighboring barrels through polarization toward the center of the home barrel.

$A$

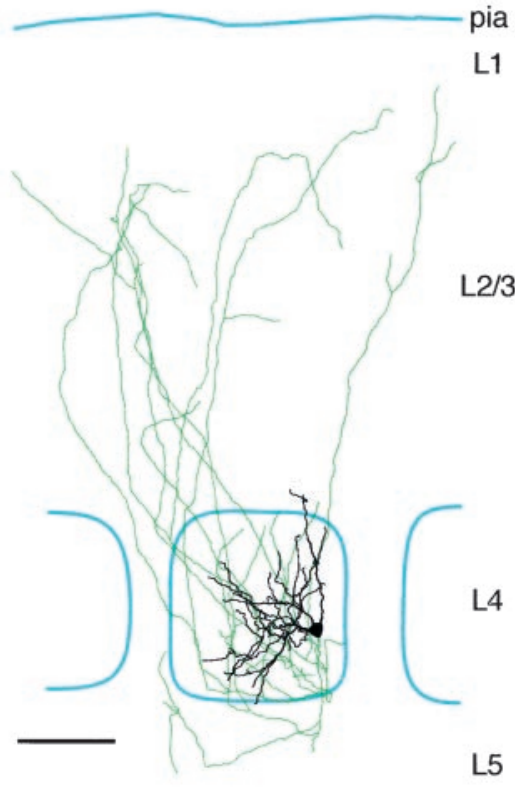

B

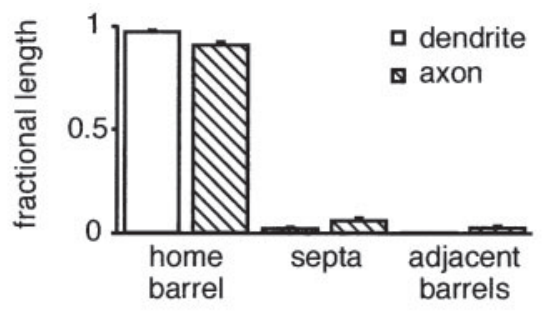

C

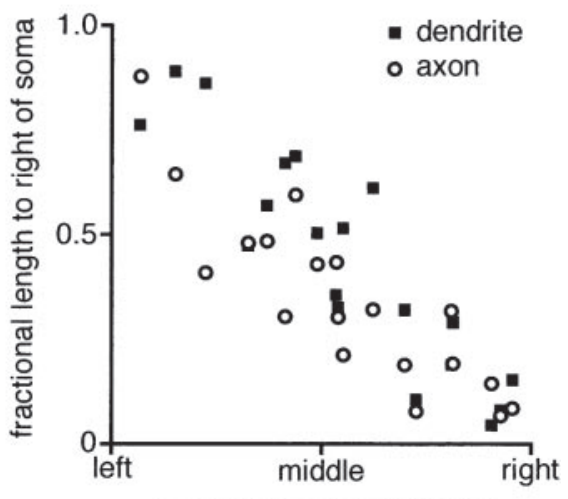

location of soma in barrel

\section{Excitatory neuronal circuits within layer 4 are functionally confined to a single barrel}

To gain an appreciation of the functional connectivity of the large-scale neuronal network we investigated the spatial extent of fEPSPs in layer 4 evoked by local stimulation within a single barrel (Fig. 5). The recording pipette was moved to different locations, both within the barrel being stimulated and in adjacent barrels, while stimulation was kept constant. Evoked fEPSPs were recorded and plotted as a function of the recording position (Fig. $5 A-C$ ). As the recording pipette was moved away from the stimulation electrode, the amplitude of the evoked fEPSP gradually decreased. However, an abrupt decrease in the fEPSP amplitude was observed as the position of recording pipette traversed a barrel boundary entering the neighboring barrel. To quantify this effect, the edge of a barrel was defined at the half-maximal intensity of the bright-field image of the barrel being stimulated with the peak representing the septum dividing neighboring barrels (Fig. 5D). Alignment of normalized field potentials as a function of distance from the barrel edge allows the pooling of data from different experiments $(n=7)$.
At the edge of the barrel there is a sharp drop in evoked fEPSPs, which is detected by a fit of a combined sigmoidal and linear function with all parameters free for optimization by a least squares fitting routine (Fig. $5 E$ ). The half-maximal point of the fitted sigmoidal function was $11 \mu \mathrm{m}$ outside the stimulated barrel with a sharp transition. Synaptic transmission under these conditions thus appears to be restricted to a single barrel.

From the anatomical data and the spatial extent of the fEPSP responses, it would thus seem reasonable to expect that neurons with somata in different barrels would not be synaptically connected. To address this, many pairs of excitatory neurons were recorded from, some with somata in neighboring barrels and some with somata in the same barrel. Attention was paid to ensure that the distance between the somata of the neurons being recorded from was always $<200 \mu \mathrm{m}$ with approximately equal distances between the pairs of neurons in the same barrel and those in neighboring barrels (somata of neurons recorded in the same barrel were separated by $98 \pm 47 \mu \mathrm{m}$, whereas somata of neurons in adjacent barrels were separated by $137 \pm 30 \mu \mathrm{m})$. Action 


\section{A}
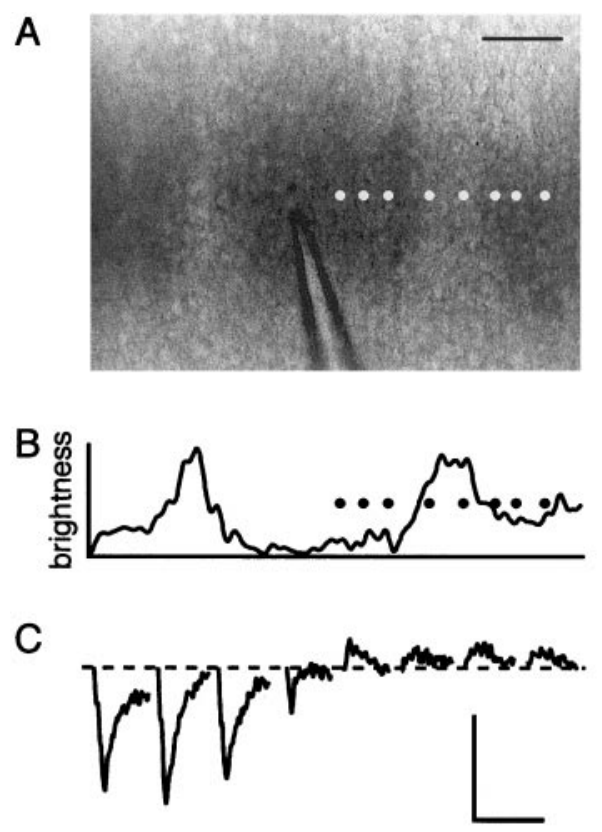

D

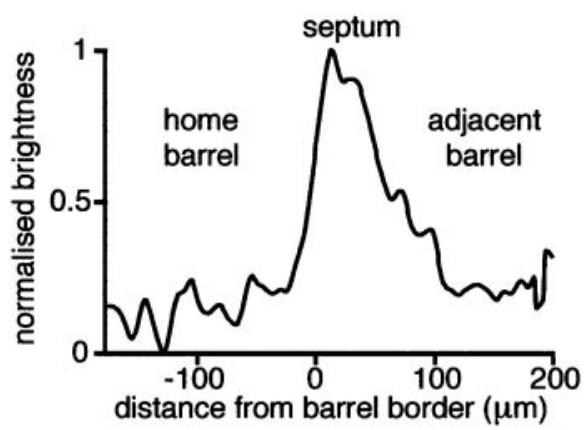

E

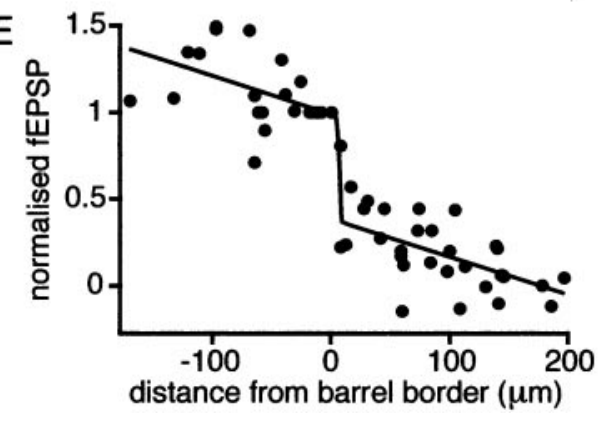

Figure 5. fEPSPs are attenuated at the barrel border. $A$, Photograph of layer 4 barrels with glass stimulation pipette in the central barrel. The various positions of the recording electrode are indicated by the white dots. Scale bar, $100 \mu \mathrm{m}$. $B$, The curve is a plot of pixel brightness across the barrels in the photograph above and indicates quantitatively how the barrels are separated by lighter regions of septum. The black dots again represent the locations where fEPSPs were recorded. $C$, Evoked fEPSPs recorded at the different positions shown above with the left to right order preserved. Relatively small responses are recorded in positions outside of the barrel where the stimulation electrode is located. $D$, The average brightness of pixels across the region of layer 4 where the field recordings were made defines the barrel border and septum for the entire set of experiments. The border of the barrel is defined as the location where the brightness is halfmaximal. E, The amplitudes of field responses recorded from many experiments at many positions were normalized to the value at the edge of the barrel and plotted against distance from the border of the barrel. The superimposed curve is the best fit (least squares method) of a summation of a linear and a sigmoidal function with all parameters held free. The fit suggests that fEPSPs are attenuated at the barrel boundary.

A1

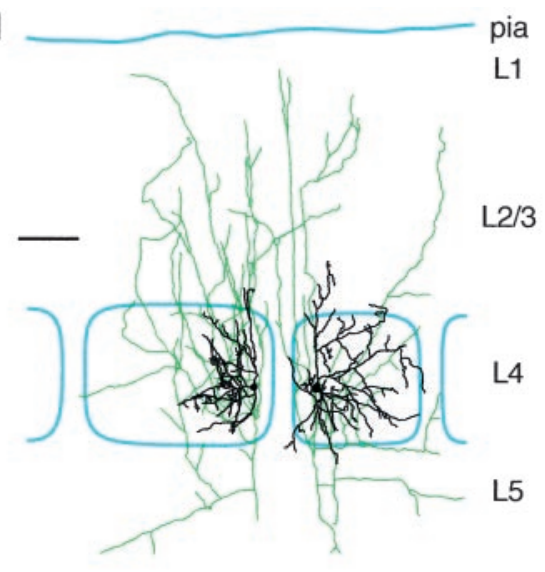

B1

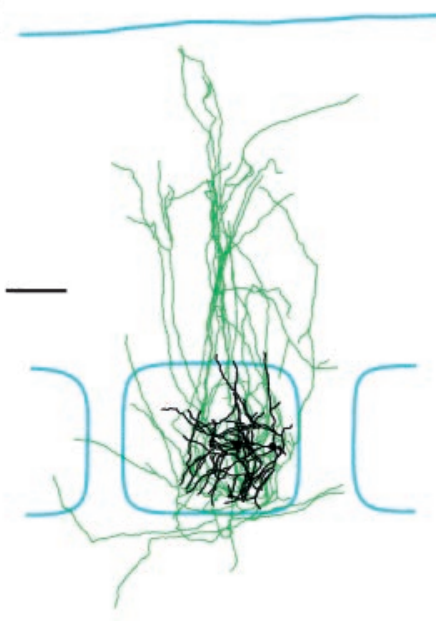

L1

L2/3
A2
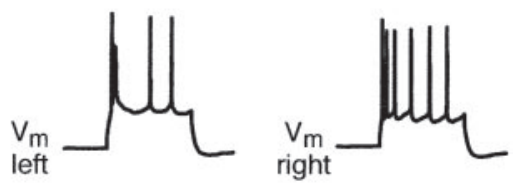

A3
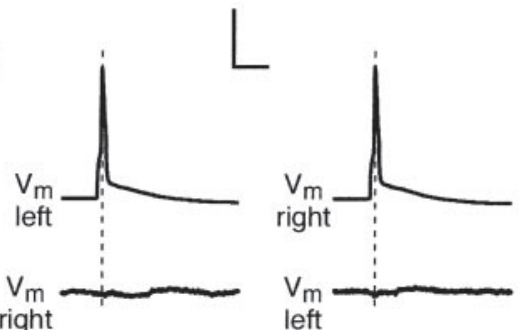

B2

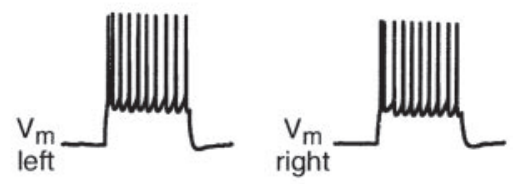

B3

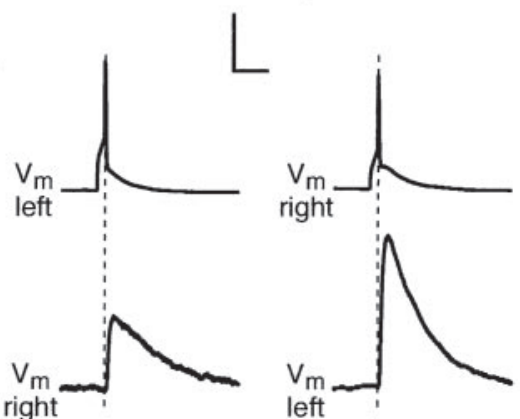

Scale bars: $A 1, B 1,100 \mu \mathrm{m}$. Calibration: $A 2$, trace) or $1 \mathrm{mV}$ (bottom trace), $10 \mathrm{msec}$.

potentials were evoked to test for connections (Fig. 6). Approximately one-fifth of the neuron pairs tested were synaptically connected (21 of 103 cell pairs). Except in one case, all of these neurons had their somata located in the same barrel as identified by cytochrome $\mathrm{C}$ staining and/or bright-field microscopy. The mean unitary EPSP (uEPSP) amplitude of the connections was $0.93 \pm 0.2$ $\mathrm{mV}$ with a range from 0.12 to $3.3 \mathrm{mV}$. The single connection found between two neighboring barrels was well within the normal range, although smaller than the average with an amplitude of $0.43 \mathrm{mV}$. In general, the axonal and dendritic trees of neurons with somata 
Figure 7. Neurons are highly connected within a barrel but not with adjacent barrels. $A$, Photograph of a slice showing barrels divided by a septum. The postsynaptic whole-cell recording pipette is visible, located close to the edge of the left-hand barrel. Square symbols indicate the locations of cells tested using the loose-patch technique that were found to be synaptically connected to the postsynaptic neuron. Open circles indicate the locations of cells that were not connected to the postsynaptic cell. The seven cells that evoked responses in the postsynaptic cell were located either in the same barrel as the postsynaptic cell or in the near half of the septum. Scale bar, $100 \mu \mathrm{m} . B$, The responses recorded in the postsynaptic cell in response to loose-patch evoked action potentials of the cells shown above. Traces in bold show the EPSPs evoked by action potentials of the connected neurons, whereas the thin traces show the absence of responses evoked by stimulation of neurons without connections to the postsynaptic cell. An example of an action potential evoked by loose-patch stimulation of a presynaptic cell is shown below. Calibration: $0.2 \mathrm{mV}$ for postsynaptic potentials, $10 \mathrm{mV}$ for presynaptic potentials, 5 msec for horizontal scale bar. $C$, Summary of locations of cells that were connected (squares) or not (circles) to the postsynaptic cells in the entire set of these rons are found to lie inside the same barrel as the location of the postsynaptic cell, which on average was located $25 \mu \mathrm{m}$ left of the barrel border. $D$, The fraction of connected cells is strongly dependent on location. Approximately one-third of the cells tested within $300 \mu \mathrm{m}$ of the postsynaptic cell within the same barrel are connected to a given postsynaptic target cell. Cells from the adjacent barrel make very few synaptic connections. The averaged observed brightness curve defining the home barrel, septum and adjacent barrel in these experiments is superimposed on the connectivity data points. $E$, The frequency of connection strengths observed for neurons in the same barrel. A wide distribution of EPSP amplitudes is observed with many small amplitude connections and a few large connections. Along with the spatial constraint imposed by the confines of the barrel, this distribution of connection strengths defines the excitatory neuronal network of layer 4 . F, A schematic diagram illustrating the connectivity observed in the experiments. Each barrel is shown to have neurons that are synaptically connected to each other within the barrel but not to adjacent barrels.
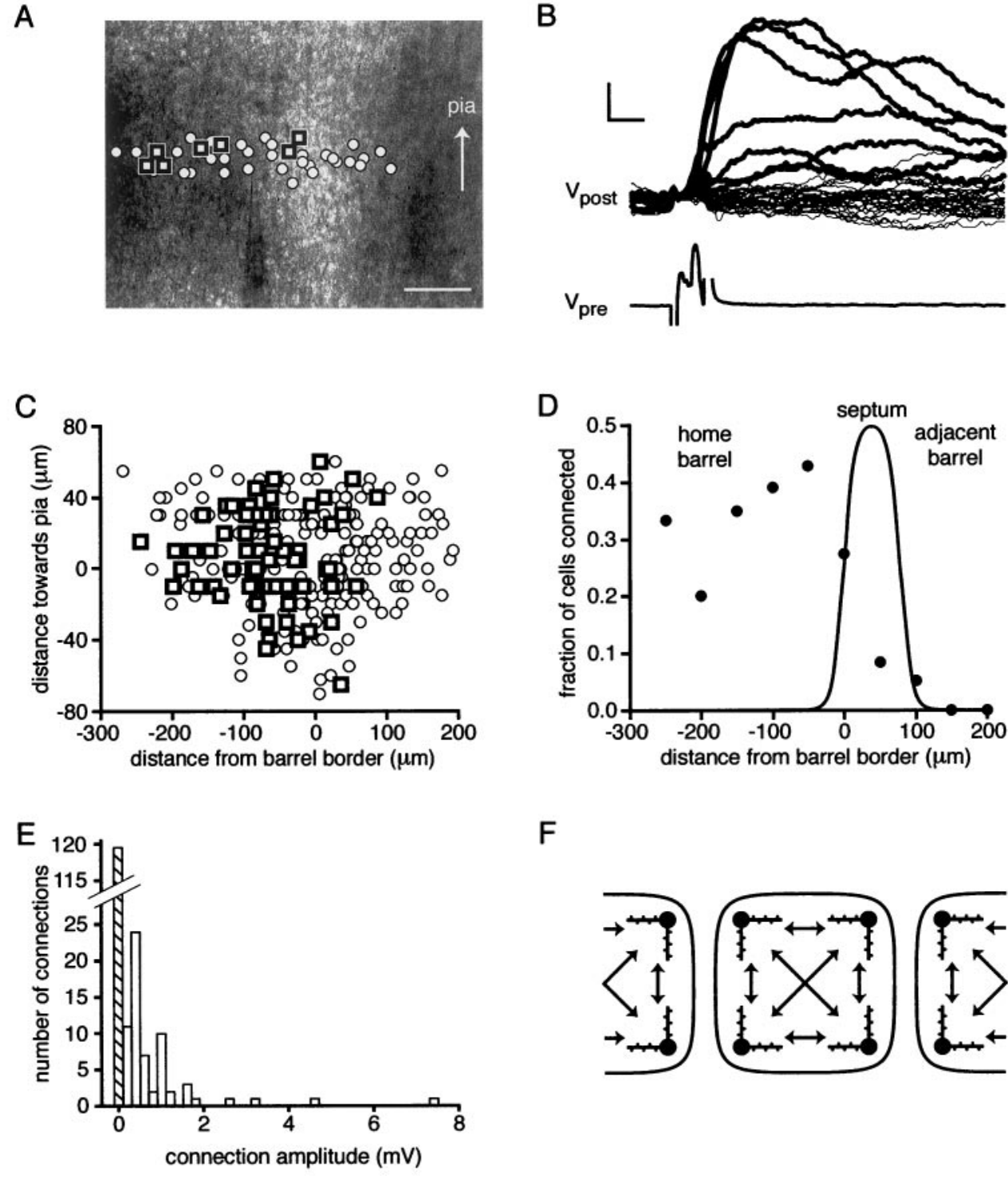

$\mathrm{F}$

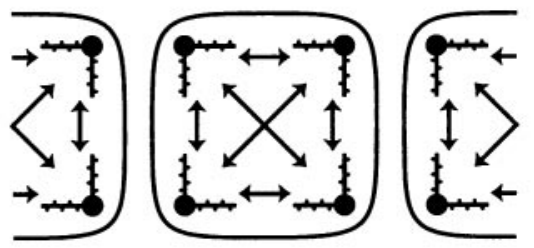
experiments. Most of the connected neu-

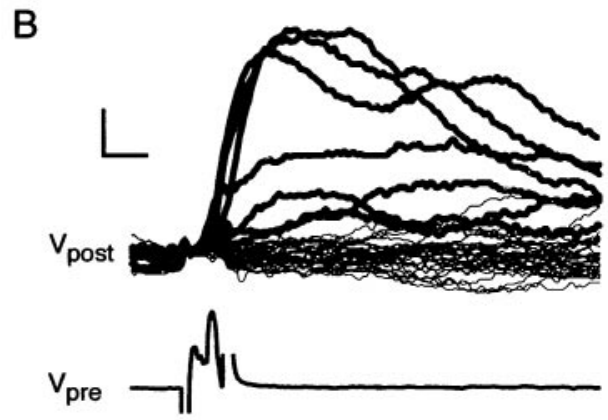

located in different barrels showed little overlap and at a functional level were not synaptically connected.

To investigate the connectivity of the excitatory neuronal network in a more systematic way, we tested for synaptic connections between many different presynaptic neurons and a single postsynaptic target cell. Whole-cell recordings were established with a postsynaptic target neuron close to the border of a barrel, with quantification of barrel boundaries as described earlier using the half-maximal pixel intensities. Using loose-patch stimulation, candidate presynaptic neurons were tested, and the location of each cell relative to the target cell and the barrel boundaries were measured. In a single experiment between 20 and 30 presynaptic cells were tested, with care being taken to test equal numbers of neurons at similar distances on either side of the postsynaptic target cell (Fig. $7 A, B$ ). To evaluate whether a pair of cells was connected, 30 action potentials were evoked in the presynaptic cell, and the average EPSP in the postsynaptic cell was obtained. A pair of neurons was classified as connected if an average peak response of $>0.1 \mathrm{mV}$ was evoked with a short latency (beginning of response within the $5 \mathrm{msec}$ presynaptic current injection period). Correlating the position of the tested presynaptic neurons with the barrel showed that most connected neurons are located within the same barrel as the postsynaptic neuron, and very few connections were found between neighboring barrels (Fig. 7A,B). Alignment of data from all experiments $(n=11)$ such that the origin occurs at the barrel border indicates that this is a robust observation (Fig. 7C). There is a sharp drop in connectivity as presynaptic neurons with somata located in the septum are tested, and very few connections can be found between neurons in different adjacent barrels. Within the barrel connectivity appears to be very high with $36 \%$ of the neurons (52 of 146) within $300 \mu \mathrm{m}$ of the target neuron being connected (Fig. 7D). This is in good agreement with the estimate of $31 \%$ connectivity reported by Feldmeyer et al. (1999). The vast majority of synaptically connected neurons are thus found to be located within the same barrel with relatively little communication between barrels at the level of layer 4 , as schematically illustrated in Figure $7 F$.

In addition to the spatial information regarding connectivity, these experiments also yield functional data concerning the amplitude of the connections. The distribution of connection amplitudes within a barrel (Fig. $7 E$ ) is far from Gaussian, showing many small amplitude connections with a few much larger connections in agreement with previous data (Feldmeyer et al., 1999). There were no strong correlations between the separation of the presynaptic and the postsynaptic neurons with the uEPSP amplitude or the connection probability within a barrel over the first $300 \mu \mathrm{m}$ inves- 
tigated. This suggests that a barrel may form an irreducible computational unit with little spatial segregation.

\section{DISCUSSION}

The anatomical and functional data presented here at both a large-scale level (DiI diffusion and fEPSP recordings) and at the level of individual neurons (reconstruction of neuronal arbors and electrophysiological analysis of synaptic connectivity) provide strong evidence for functionally independent excitatory neuronal networks within each barrel as defined by cytochrome $\mathrm{C}$ staining or bright-field microscopy.

\section{Neuronal circuitry underlying the whisker-evoked receptive field}

The barrel cortex gains its physiological significance from the observation that each barrel corresponds both functionally and anatomically to an isomorphic sensory whisker. Extracellular recordings of neuronal activity have revealed receptive fields dominated by a single whisker isomorphic to the cortical barrel (Welker, 1976; Simons, 1978; Armstrong-James and Fox, 1987). Although a principal whisker can often be clearly defined for cortical neurons it is also clear that neurons in somatosensory cortex can respond to more than a single whisker. Layer 4 neurons have the sharpest receptive fields, but even for these neurons whiskers surrounding the principal whisker can evoke EPSPs (Moore and Nelson, 1998; Zhu and Connors, 1999). In general these surround whisker responses have reduced amplitude and increased latency compared to the principal whisker (Armstrong-James et al., 1992). One possible mechanism that has been suggested to underlie responses to surround whiskers involves local cortical circuits. Although this may well provide an important contribution to multiwhisker responses in layer $2 / 3$ and layer 5 , the data presented in this paper show that layer 4 barrels are anatomically and functionally independent. The excitatory responses to surround whiskers recorded in layer 4 might thus be attributed to earlier stages of the signaling pathway. In agreement with this hypothesis, a recent in vivo study analyzing the effects on vibrissa responses of ablating the neighboring neocortical barrel found little reduction in the amplitude of responses to surround whiskers (Goldreich et al., 1999). Furthermore, multiwhisker responses have been detected in thalamocortical neurons of the ventral posterior nucleus (Ito, 1988; Simons and Carvell, 1989; Armstrong-James and Callahan, 1991; Diamond et al., 1992). Because these thalamocortical neurons project into the barrels of layer 4 , it is perhaps not unexpected that large receptive fields can be recorded within layer 4 , although at present it is unclear whether this is adequate to account for the response properties quantitatively. In addition, layer 4 neurons receive substantial input from layer 6 (Stratford et al., 1996), and it would interesting to investigate the underlying neuronal circuitry determining whether the dendrites and axons of these neurons respect barrel boundaries.

\section{Determinants of connectivity}

The boundaries of the barrel appear to provide a simple rule for connectivity in layer 4 barrel cortex. Neurons within a barrel can be synaptically connected, but because both dendritic and axonal arbors are confined to the home barrel, there are few connections to neighboring barrels. Within a barrel there is a high degree of connectivity, which does not appear to depend strongly on distance between the neurons, at least over the first $300 \mu \mathrm{m}$ investigated in this study (Fig. 7D). Barrels of $<300 \mu \mathrm{m}$ diameter may thus represent spatially homogenous processing units. Two further pieces of evidence support this notion. First, there was no correlation between amplitudes of uEPSPs and the separation of the presynaptic and postsynaptic neurons within the same barrel. Second, the horizontal span of both axonal and dendritic arborizations derived from our anatomical reconstructions extend across a large fraction of the barrel diameter. The mean horizontal span within layer 4 of axonal processes was $262 \pm 86 \mu \mathrm{m}$ and of dendritic processes was $181 \pm 40 \mu \mathrm{m}$ in barrels of mean diameter $240 \pm 59$ $\mu \mathrm{m}$. It thus appears that there is no obvious spatial segregation of neuronal circuitry within a barrel, although further investigations should address the matter specifically with respect to the large barrels of the posterior medial subfield.

The data suggest that there is a very broad range of uEPSP connection strengths. The distribution of amplitudes indicates that there are a few large connections, but the majority are under well under $2 \mathrm{mV}$ in peak amplitude. Such a distribution is unlikely to be formed by a process of random connectivity, which would result in connectivity distributions closer to Gaussian or decaying exponential. Such functions are able to fit the observed connectivity for small amplitudes $(<2 \mathrm{mV})$, but they fail to account for the large amplitude EPSP connections. That the distribution of connection amplitudes is far from random suggests that rules may exist for determining the connectivity of the excitatory neuronal network within a layer 4 barrel.

Recently, Egger et al. (1999) described a form of long-term depression found between excitatory neurons of layer 4, which would decrease the efficacy of a synaptic connection if both presynaptic and postsynaptic neurons are highly active simultaneously. Such a process will act to destabilize large connections and could account for the bias to small connections. As yet no mechanism for increasing the synaptic strength of connections between layer 4 neurons has been identified, and in particular Egger et al. (1999) also reported that there does not appear to be any NMDA receptor-dependent synaptic potentiation within the mature layer 4. Perhaps, in analogy to the thalamocortical synapses, there is a critical period for NMDA receptor-dependent potentiation such that rats $>1$-week-old do not express this type of plasticity (Crair and Malenka, 1995). There is thus a need to explore the development of layer 4 barrel cortex more closely to investigate what determinants of connectivity are active as the neuronal circuitry is established.

Sensory deprivation provides a method for the analysis of activity-dependent reorganization of the somatosensory signaling pathway. Although the developing layer 4 is clearly able to undergo plastic changes in response to deprivation of sensory input (Van der Loos and Woolsey, 1973; Wong-Riley and Welt, 1980; Schlaggar et al., 1993) it is at present unclear to what extent the neuronal network of layer 4 in the adult can be rearranged (Diamond et al., 1994; Glazewski and Fox, 1996). Our understanding of the determinants of connectivity would benefit from a detailed analysis of what changes occur at both a morphological and physiological level to the neuronal network after sensory deprivation. The description of the undeprived excitatory neuronal network presented here provides a reference point for studying plastic changes induced by alteration in sensory input.

The simplest interpretation of the connectivity data of the layer 4 excitatory neuronal network within barrel would assume that the connection between any two neurons can be chosen randomly from the observed distribution. However, it is possible that the rules of development and long-term plasticity lead to networks far from the stochastic situation. Although there is no obvious spatial segregation of neuronal networks within a barrel, there could in fact be functional subnetworks of neurons, which the present set of experiments would not have observed. Further experiments with simultaneous recordings from many neurons will be necessary to address this important issue.

\section{REFERENCES}

Agmon A, Connors BW (1991) Thalamocortical responses of mouse somatosensory (barrel) cortex in vitro. Neuroscience 41:365-380.

Armstrong-James M, Callahan CA (1991) Thalamocortical processing of vibrissal information in the rat. II Spatiotemporal convergence in the thalamic ventroposterior medial nucleus (VPM) and its relevance to generation of receptive fields of S1 cortical "barrel" neurons. J Comp Neurol 303:211-224.

Armstrong-James M, Fox K (1987) Spatiotemporal convergence and divergence in the rat SI "barrel" cortex. J Comp Neurol 263:265-281.

Armstrong-James M, Fox K, Das-Gupta A (1992) Flow of excitation within rat barrel cortex on striking a single vibrissa. J Neurophysiol $68: 1345-1358$ 
Crair MC, Malenka RC (1995) A critical period for long-term potentiation at thalamocortical synapses. Nature 375:325-328.

Diamond ME, Armstrong-James M, Ebner FF (1992) Somatic sensory responses in the rostral sector of the posterior group (POm) and in the ventral posterior medial nucleus (VPM) of the rat thalamus. J Comp Neurol 318:462-476.

Diamond ME, Huang W, Ebner FF (1994) Laminar comparison of somatosensory cortical plasticity. Science 265:1885-1888.

Egger V, Feldmeyer D, Sakmann B (1999) Coincidence detection and changes of synaptic efficacy in spiny stellate neurons in rat barrel cortex. Nat Neurosci 2:1098-1105.

Feldmeyer D, Egger V, Lübke J, Sakmann B (1999) Reliable synaptic connections between pairs of excitatory layer 4 neurones within a single "barrel" of developing rat somatosensory cortex. J Physiol (Lond) 521:169-190.

Finnerty GT, Roberts LS, Connors BW (1999) Sensory experience modifies the short-term dynamics of neocortical synapses. Nature 400:367-71.

Glazewski S, Fox K (1996) Time course of experience-dependent synaptic potentiation and depression in barrel cortex of adolescent rats. J Neurophysiol 75:1714-1729.

Goldreich D, Kyriazi HT, Simons DJ (1999) Functional independence of layer IV barrel in rodent somatosensory cortex. J Neurophysiol 82:1311-1316.

Harris RM, Woolsey TA (1983) Computer-assisted analyses of barrel neuron axons and their putative synaptic contacts. J Comp Neurol 220:63-79.

Ito M (1988) Response properties and topography of vibrissa-sensitive VPM neurons in the rat. J Neurophysiol 60:1181-1197.

Kim U, Ebner FF (1999) Barrels and septa: separate circuits in rat barrel field cortex. J Comp Neurol 408:489-505.

Lübke J, Egger V, Sakmann B, Feldmeyer D (2000) Columnar organization of dendrites and axons of single and synaptically coupled excitatory spiny neurons in layer 4 of the rat barrel cortex. J Neurosci 20:5300-5311.
Moore CI, Nelson SB (1998) Spatio-temporal subthreshold receptive fields in the vibrissa representation of rat primary somatosensory cortex. J Neurophysiol 80:2882-2892.

Polley DB, Chen-Bee CH, Frostig RD (1999) Two directions of plasticity in the sensory-deprived adult cortex. Neuron 24:623-637.

Schlaggar BL, Fox K, O'Leary DDM (1993) Postsynaptic control of plasticity in developing somatosensory cortex. Nature 364:623-626.

Simons DJ (1978) Response properties of vibrissa units in the rat SI somatosensory neocortex. J Neurophysiol 41:798-820.

Simons DJ, Carvell GE (1989) Thalamocortical response transformation in the rat vibrissa/barrel system. J Neurophysiol 61:311-330.

Stratford KJ, Tarczy-Hornoch K, Martin KAC, Bannister NJ, Jack JJB (1996) Excitatory synaptic inputs to spiny stellate cells in cat visual cortex. Nature 382:258-261.

Van der Loos H, Woolsey TA (1973) Somatosensory cortex: structural alterations following early injury to sense organs. Science 179:395-398.

Welker C (1976) Receptive fields of barrels in the somatosensory neocortex of the rat. J Comp Neurol 166:173-190.

Wong-Riley MTT, Welt C (1980) Histochemical changes in cytochrome oxidase of cortical barrels after vibrissal removal in neonatal and adult mice. Proc Natl Acad Sci USA 77:2333-2337.

Woolsey TA, Van der Loos H (1970) The structural organisation of layer IV in the somatosensory region (SI) of the mouse cerebral cortex: the description of a cortical field composed of discrete cytoarchitectonic units. Brain Res 17:205-242.

Woolsey TA, Dierker ML, Wann DF (1975) Mouse SmI cortex: qualitative and quantitative classification of Golgi-impregnated barrel neurons. Proc Natl Acad Sci USA 72:2165-2169.

Zhu JJ, Connors BW (1999) Intrinsic firing patterns and whiskers-evoked synaptic responses of neurons in the rat barrel cortex. J Neurophysiol 81:1171-1183. 